\title{
Analysis of Rice Granules using Image Processing and Neural Network Pattern Recognition Tool
}

\author{
Abirami. S \\ Assistant Professor \\ Saranathan College Of \\ Engineering \\ Panjappur, Trichy - 620012 \\ India
}

\author{
Neelamegam. P \\ Professor \\ SASTRA University \\ Triumalaisamudram \\ Thanjavur, India-613401
}

\author{
Kala. $\mathrm{H}$ \\ Assistant Professor \\ Saranathan College Of \\ Engineering \\ Panjappur, Trichy - 620012 \\ India
}

\begin{abstract}
In foodstuff trade, grading of coarse food resources is essential because samples of stuffs are subjected to adulteration. In the precedent, foodstuffs in the appearance of granules were conceded through sieves or supplementary mechanical way for grading purposes. In this manuscript, investigation is performed on basmati rice granules; to appraise the act via image processing and Neural Network Pattern Recognition Tool which is implemented based on the features extracted from rice granules for categorization grades of granules. Digital imaging is acknowledged as a proficient system, to haul out the features from rice granules in a non-contact mode. Images are acquired for rice using camera. Image Pre-processing techniques, Adaptive thresholding, Canny edge detection, Feature extraction are the checks that are performed on the acquired image using image processing method through Open source Computer Vision (Open CV) which is a library of functions that aids image processing in real time. The morphological features extracted from the image are given to Neural Network Pattern Recognition Tool. This effort has been prepared to categorize the appropriate quality category for a specified rice sample based on its parameters. The performance of image processing condensed the time of action and enhanced the crop identification significantly.
\end{abstract}

\section{Keywords}

Open CV, Neural Network Pattern Recognition Tool, Adaptive Thresholding, Canny Edge Detection, Morphological features.

\section{INTRODUCTION}

Quality of grains is a vital prerequisite for today's souk, to shelter the patrons from second-rated foodstuffs. Some traders dishonorably discharge poor quality products to the shopper market. Because of such practices there are numerous low-grade quality grains arriving to the marketplace day by day. These grains consists of several impurities like stones, weed seeds, chaff, damaged seeds, more broken granules etc. This is frequently seen today in rice deal where rice in stumpy quality is sold without being noticed. Conversely, there is no opportune scheme to recognize these second-rated quality grains in the market. Therefore, this has become a stern crisis for mutually the purchaser and the regime. It is trouble-free for the purchaser fortification influence to abstain from executing the duties as the categorization of manufactured goods such as rice with reverence to the foreign substances, broken granules etc. is indistinct. Therefore, it is requisite to survey the likelihood of using technology for an appropriate elucidation. The exactness of quality scrutiny via human assessment scheme is different from person to person according to the inspectors' physical status such as working hassle, point of view and fidelity for traders. Also, the understanding and skill of inspectors are obligatory to precisely execute this appraisal progression.
Substantial work for classifying and identifying varieties of grains has been reported. B.S. Anami et al., [1] described a method for gradation and classification of different grains such as wheat, Bengal gram, groundnut, etc. An artificial neural network approach is used in the Identification and classification of the bulk grain samples by N.S. Visen et.al, [2]. Harlick, et.al., [3] has presented a paper on classification of image using textural features. This work is done based on gray-tone spatial dependencies for easily computable textural features. LIU zhaoyan et al., [4] projected his ideas on Identification of different varieties of rice grains using neural network and image processing. They used an algorithm of digital imaging based on morphological and color features of different rice varieties. By using image analysis techniques M.A. Shahin and S.J. Symons [5] automated the manual sieving procedure. Using flatbed scanner, J.Paliwal et al., [6] performed a research for both bulk and single seed images. N.S. Visen et al., [2] developed and optimized a technique by extracting the morphological, texture, and color features using images of single grains for discriminating various types of grains. Identifying the food grains and evaluating its quality using pattern classification is done by Sanjivani Shantaiyai, et al., [7]. H.Rautio and O.Silvn [8] carried out research to verify the normal grain size and classified using morphology and texture features. This paper presents classification of grades of Basmati rice granules using the novel system Image Processing and Neural Network Pattern Recognition Tool.

\section{MATERIALS AND METHODOLOGY}

The samples of Basmati rice grains were collected from stores and a Nikon camera is used to attain and trace the imagery for rice granules of diverse sizes. The camera is mounted on a plunk which provides vertical movement. When the camera is fixed, distance between the lens and the sample table with uniform background, is $30 \mathrm{~cm}$. The back ground is black. The uniform intensity lighting on the sample table is provided. Inside the field of view, the grains were arranged in random point of reference and arrangement. Images were stored in jpg format and diverse parameters of rice were extracted from the image for added scrutiny. With the parameters interpreted we set up a Neural Network system using Scaled Conjugate Gradient for grading of rice granules.

\section{IMAGE VISION}

Image analysis involves the process of differentiating the granules from the background and extracting quantitative information, which is used further for decision making process.

\subsection{Image Acquisition and Smoothing}

The first step in image processing is Image Acquisition. Acquirement of an image is done by using Nikon camera beneath 
homogeneous lighting arrangement. Customary measures are applied for improving the value of an image through Preprocessing techniques. In this paper, smoothing is done using Median Filters. Median filtering is extensively used in digital imaging since; it conserves the ends of the image during noise exclusion [9]. Speckle noise and salt and pepper noise are which with, median filters are predominantly effectual [10]. Using median filter the noise in the input gray color image is removed. Fig 1 and Fig 2 shows the acquired and the smoothened image.

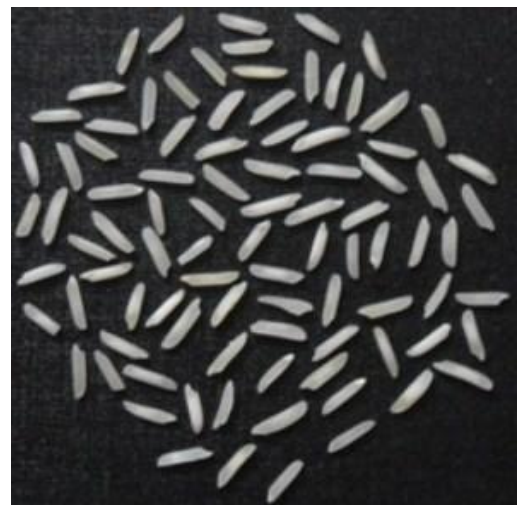

Fig 1: Acquired Image

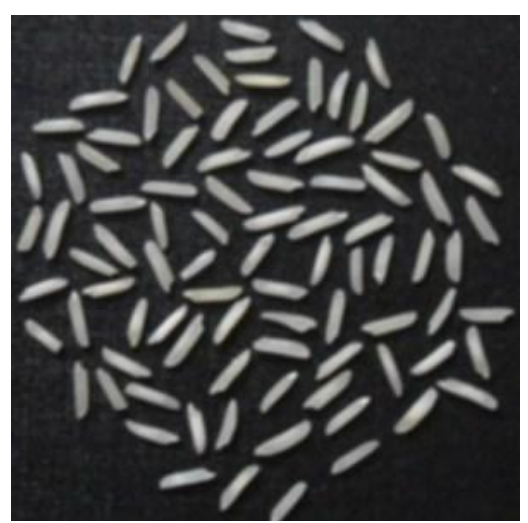

Fig 2: Smoothing Image

\subsection{Thresholding}

The subsequent step is to segment an image which is one of the imperative stages in image analysis. The accurateness of this action is greatly reliant on consequently extracted records. Sonka et al., [11]; Sun [12], projected that Segmentation is attained by three techniques such as Edge segmentation, Region segmentation and Thresholding. In this manuscript we execute segmentation through adaptive thresholding, and recognition of edges through canny edge detection.

Thresholding is a practice based on assimilation of light in their surfaces to exemplify the regions of the image. Threshold is to detach the regions in an image with reverence to the stuffs, which is to be analyzed. This partition is based on the discrepancy of intensity between the object pixels and the background pixels. In our effort to execute thresholding, adaptive thresholding system is implemented. Once we have appropriately estranged the essential pixels, we can set them with a firm value to categorize them (i.e. we can assign them a value of 0 (black), 255(white) or any value that suits our needs). Fig 3 shows the outcome from Adaptive thresholding.

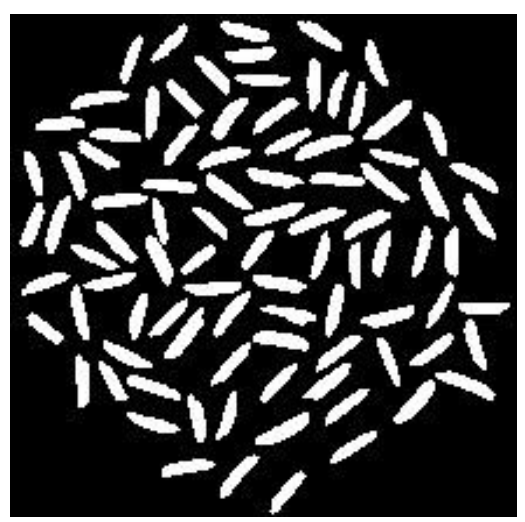

Fig 3: Threshold Image

\subsection{Canny Edge Detection}

Edge detection is based on recognition of edges by diverse edge operators. Discontinuities in color, grey level, texture, etc. are detected by edge operators.

Canny edge detector is a most favorable detector which gives finest filtered image. The gray scale image boundaries are detected by this optimal recognition process. The edges in the image are perceptible only on one occasion and forged edges are not produced owing to the clamor in the image as Canny's technique has good recognition and localization with negligible comeback. This detector has the capability to perceive feeble edges and is significantly sturdy. Canny edge detector distinguishes the edges by locating the local maxima and minima of the gradient of the intensity function [13]. The Canny edge detector blurs the images faintly, and then applies an algorithm that thins the edges to one-pixel efficiently. The upshot of canny Edge Detection is shown in the Fig 4.

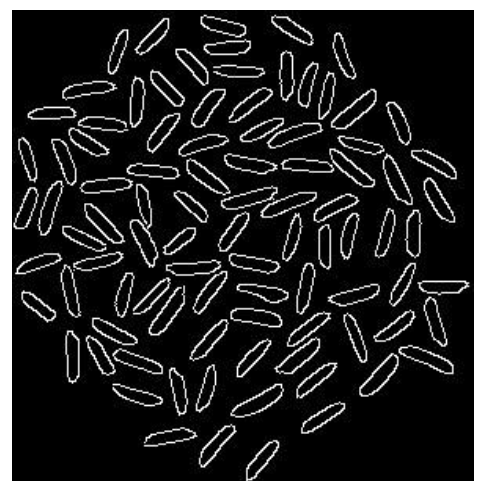

Fig 4: Canny Edge Detection

\subsection{Feature Extraction}

Extraction of quantitative information from segmented images is dealt with Feature Extraction. Object identification and classifications are performed based on diverse algorithms of morphological features. The features which were extracted from images of rice kernels are Perimeter, Area, Minor-axis Length and Major-axis Length using Contour detection which is shown in Fig 5. The collected data were then used in Neural Network Pattern Recognition system for grading of rice kernels. 


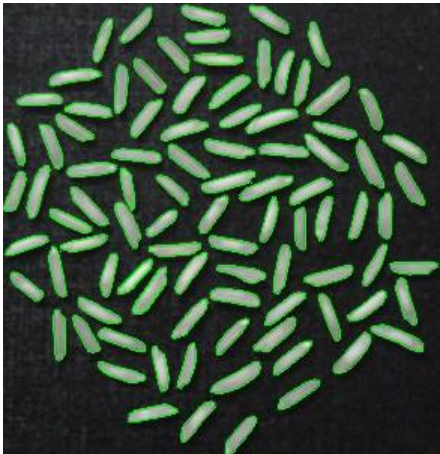

Fig 5: Contour Image

\section{GRADING USING NEURAL NETWORK PATTERN RECOGNITION TOOL}

To regiment the grading progression, labor-intensive scheme desires to be replaced with an approach which is used to steady the practice. The grading is determined in this manuscript using neural network pattern recognition tool based on the extracted features from the rice samples. Fig 6 shows the sample datasets loaded from workspace. Fig 7 shows the neural network architecture. The neural network is built with four neurons in input layer, nine neurons in the hidden layer and three neurons in the output layer. The network used for classification is Scaled Conjugate Gradient Training.

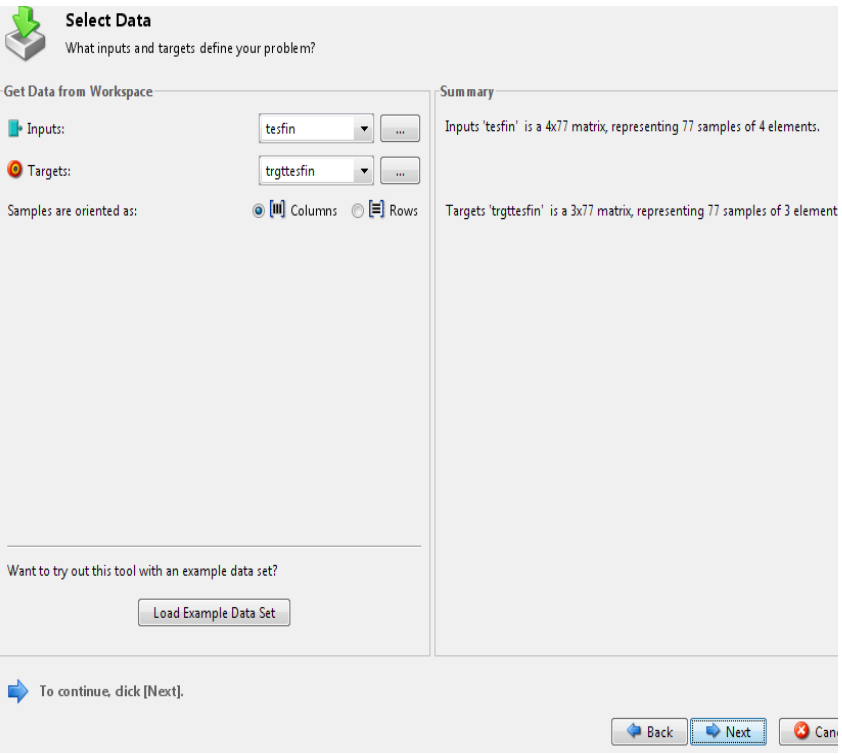

Fig 6: Sample Datas loaded

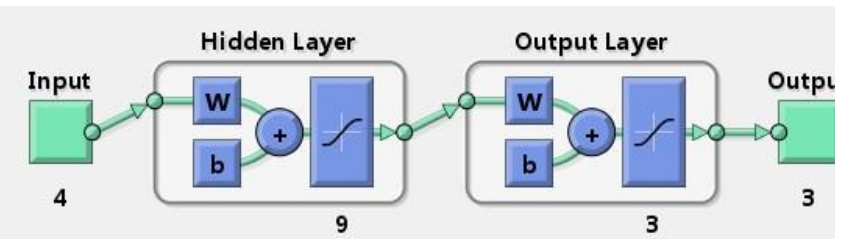

Fig 7: Neural Network Architecture

\section{RESULTS AND DISCUSSIONS}

Quality assessment of Basmati rice seeds is performed in this document by means of image processing. Calculations of perimeter, minor axis length, area, major axis length are primed for a given sample. The rice granules are graded depending on the dimension of grains present in the sample. Based on the dimensions they graded as grade 1 , grade 2 and grade 3. 53 samples were used for training, 12 samples for validation and 12 samples for testing. Fig 8 shows the classification of samples and $\%$ Mean Squared Error (MSE). Fig 9 shows the algorithms and its progression. The progress was completed in 33 epochs and the time taken to complete the iterations was 0.01 second. A Performance Plot was plotted for Epochs versus MSE which is shown in fig 10 . Best validation performance is 0.011598 which was reached at epoch 27. Fig 11 shows the Confusion matrix which shows the grading of rice granules as grade1, grade 2 and grade 3 .

Number of images taken for training and testing has been restricted due to time constrained. From the overall confusion matrix 22 samples were classified as grade 1,20 samples were graded as grade 2 and 34 samples were graded as grade 3 . When there is no overlap of grains, it is able to classify well for all the grains and the accuracy is found to be $98.7 \%$. If there is overlap of grains, the Neural Network is not able to classify correctly and in this case the accuracy is found to be $91.3 \%$.

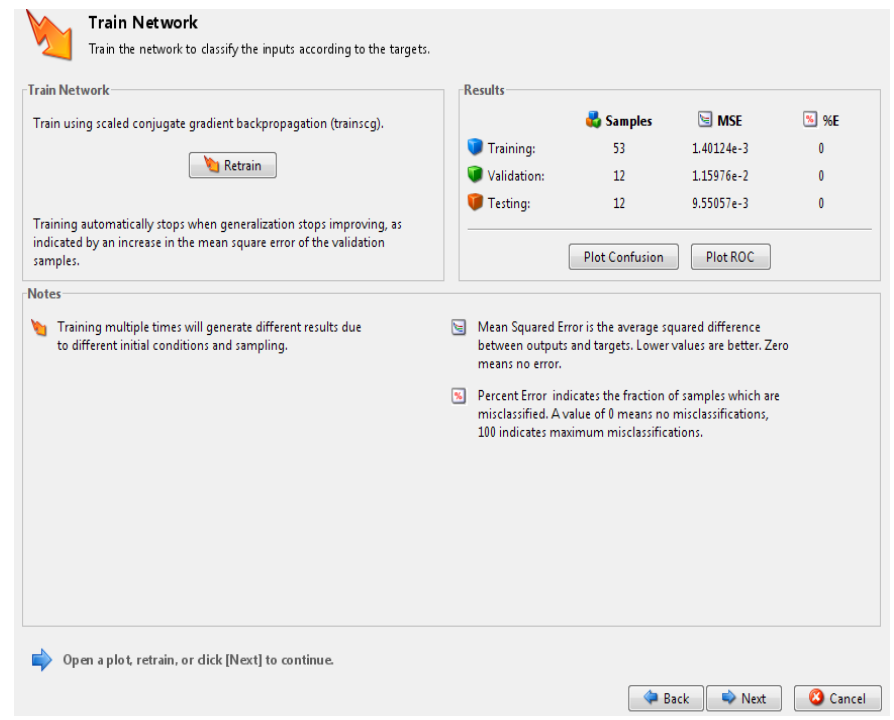

Fig 8: Samples Classification and \%MSE 


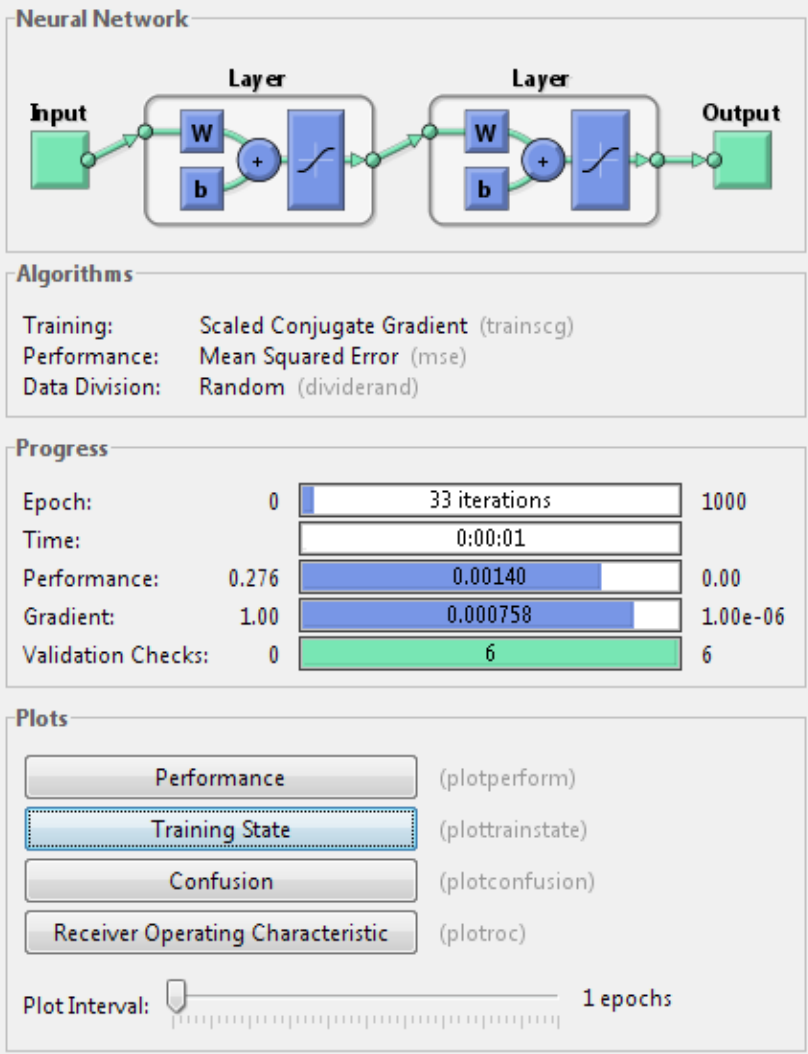

Opening Perfor mance Plo1

Stop Training 8 Cancel

Fig 9: Algorithms and Progress

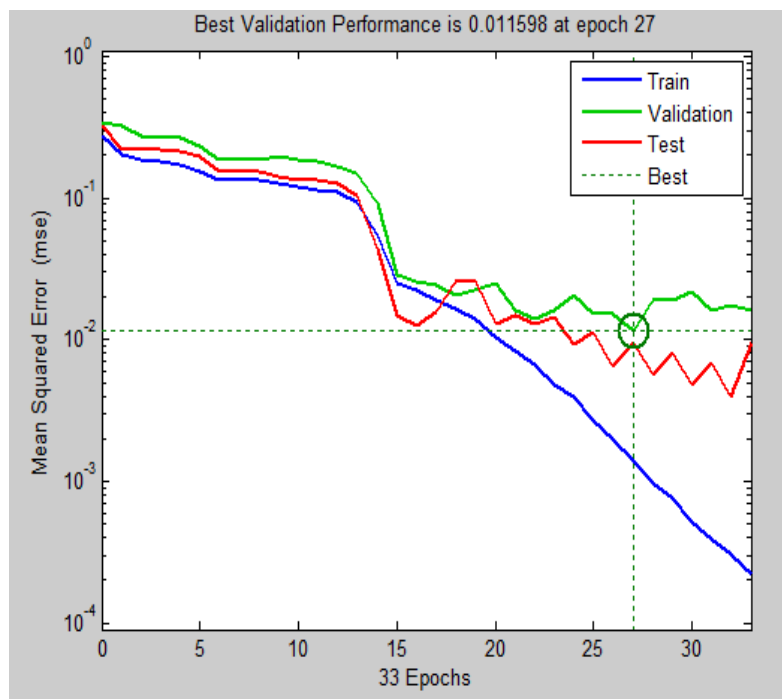

Fig 10: Performance Plot

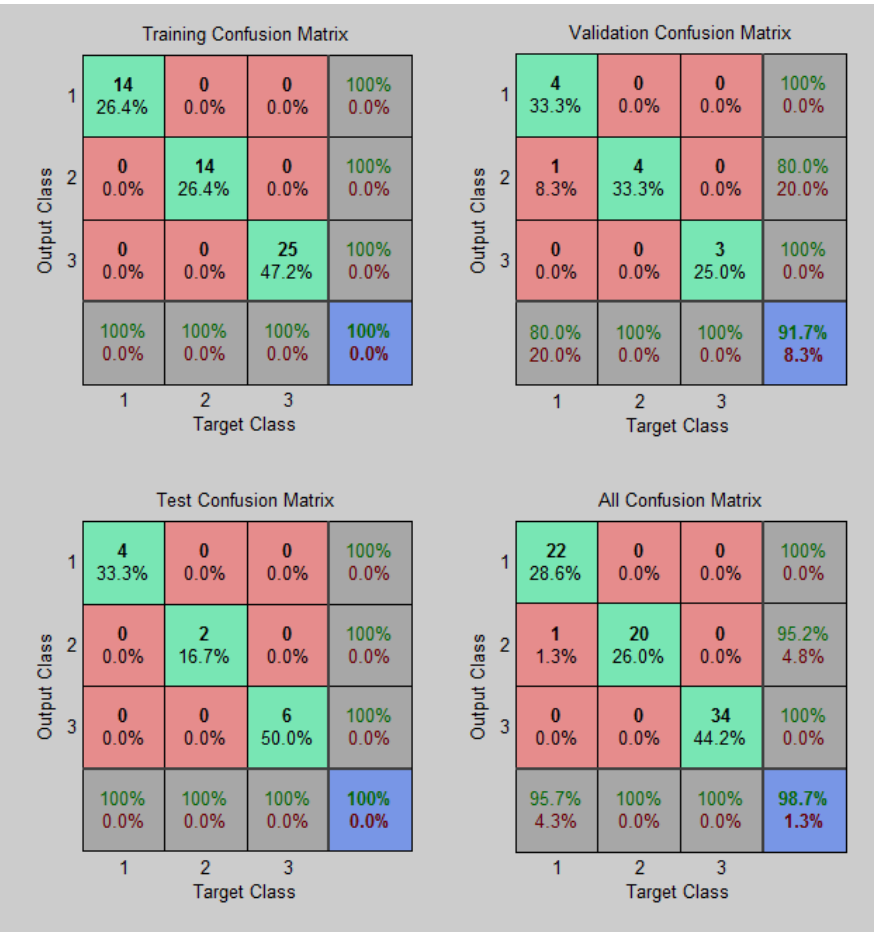

Fig 11: Classification Of Rice Granules (Confusion Matrix)

\section{CONCLUSION}

The processing of imagery and the vigilant assortment of the variety measured in this effort for extracting features from rice granules significantly abridged the intricacy of the grading problem. Neural Network Pattern Recognition Tool is lucratively applied in grading rice granules. The developed Neural Network can be adapted for grading added grains and foodstuffs as well. Scaled Conjugate Gradient Training based Neural Network is able to classify well when there is no overlapping of granules with the accuracy of $98.7 \%$.

\section{ACKNOWLEDGEMENT}

Our heartfelt gratitude to Dr.P.Neelamegam, who initiated us in opening this rice granules assignment.

\section{REFERENCES}

[1] B. S. Anami, V. Burkpalli, S. A. Angadi, and N. M. Patil, "Neural network approach for grain classification and gradation," Proceedings of the second national conference on document analysis and recognition, pp. 394-408, July 2003.

[2] N. S. Visen, J. Paliwal, D. S. Jayas, and N. D. G. White, "Image analysis of bulk grain samples using neural networks," Canadian Biosystems Engineering, vol. 46, pp. 7.11-7.18, 2004.

[3] R. M. Haralick, K. Shanmugam, and I. Dinstein, "Texture features for image classification," IEEE Trans. on Syst.,Man, and cybern, vol 6, pp. 610-621, 1973.

[4] L. Zhao yan, C. Fang, Y. Yibin, and R. Xiuqin, "Identification of rice seed varieties using neural network", Journal of Zhejiang University SCIENCE, September 2005. 
[5] M. A. Shahin and S. J. Symons, "Seed sizing from images of non-singulated grain samples", Can. BioSyst. Eng, vol. 47, 2005.

[6] J. Paliwal, M. S. Borhan and D. S. Jayas, "Classification of cereal grains using a flatbed scanner", Can Biosyst Eng, vol. 46, 2004.

[7] Sanjivani Shantaiya, Mrs.Uzma Ansari, "Identification Of Food Grains And Its Quality Using Pattern Classification," International Journal of Computer \& Communication Technology, vol 2, 2010.

[8] H. Rautio and O. Silvn, "Average Grain Size Determination using Mathematical Morphology and Texture Analysis".

[9] S. Deena Christilda, M. Prathiba, and P. Neelamegam , “ Quality Inspection of Parenteral Vials Using Digital Image Analysis," Sensors \& Transducers Journal, Vol. 145, Issue 10, pp. 130-137, October 2012.
[10] R. Deepak Prasanna, P. Neelamegam, S. Sriram, Nagarajan Raju, "Enhancement of vein patterns in hand image for biometric and biomedical application using various image enhancement techniques," Procedia Engineering, vol. 38, $1174-1185,2012$.

[11] Sonka, M, Hlavac, V, Boyle, R., "Image Processing, Analysis, and Machine Vision", PWS publishing, California, USA, 1999.

[12] D. W. Sun, "Inspecting pizza topping percentage and distribution by a computer vision method," Journal of Food Engineering, vol. 44, pp 245-249, 2000.

[13] Neelamegam. P, Abirami. S, Vishnu Priya. K, Rubalya Valantina. S., "Analysis of rice granules using Image Processing and Neural Network", in IEEE Conference on Information and Communication Technologies (IEEE), ISBN No. 978-1-4673-5759-3, pp. 879-884, 2013. 